\section{Cahiers de Narratologie}

Analyse et théorie narratives

$33 \mid 2018$

L'Art du roman chez Umberto Eco

\title{
La filature du sens ou l'en-quête dans le Nom de la rose de Umberto Eco
}

\section{Khalid Hadji}

\section{(2) OpenEdition}

\section{Journals}

Electronic version

URL: http://journals.openedition.org/narratologie/7976

DOI: 10.4000/narratologie.7976

ISSN: 1765-307X

Publisher

LIRCES

\section{Electronic reference}

Khalid Hadji, «La filature du sens ou l'en-quête dans le Nom de la rose de Umberto Eco », Cahiers de Narratologie [Online], 33 | 2018, Online since 23 July 2018, connection on 15 November 2019. URL : http://journals.openedition.org/narratologie/7976 ; DOI : 10.4000/narratologie.7976

This text was automatically generated on 15 November 2019.

Article L.111-1 du Code de la propriété intellectuelle. 


\section{La filature du sens ou l'en-quête dans le Nom de la rose de Umberto Eco}

Khalid Hadji

$1 \quad$ Notre lecture du roman d'Umberto Eco Le Nom de la rose avoue dès le titre de ce travail l'inscription de deux traditions qui président simultanément à l'écriture du roman et à l'élaboration de l'action et des attitudes du personnage central. Il s'agit de deux canons d'écriture qui sont d'un côté, le registre didactique et, par extension, la position toute philosophique du penseur rationnel et logique; de l'autre, la démarche policière de l'enquête, dont ce même personnage est investi. Nous éludons, pour les besoins de la cause, la tradition religieuse et le discours qui l'accompagne dans le façonnage de l'œuvre, parce que cela a trait à la problématique de l'esthétique romanesque en rapport avec la posture scolastique et le débat religieux du Moyen-Âge, problématique qui constituerait une digression, pour ne pas dire un hors sujet, dans le cadre de ce travail sur la configuration du sens, en rapport étroit avec la mise en récit.

2 Cette référentialité bivalente, consistant à assumer une double fonction, celle d'enseigner la réflexion rationnelle et d'enquêter sur des meurtres mystérieux, prédispose le récit à ses métamorphoses potentielles, comme si l'écriture était en train de s'effectuer avec ses ratures et ses reprises, avec le plaisir de ce qui se réalise, se réactualise en demeurant mouvement.

3 Ce processus de recomposition après une décomposition, qui a succédé à une composition, est le propre de toute activité de lecture procédant par transposition des faits de l'écriture en tant qu'ensemble de combinaisons de signes, de structures, d'idées, de l'imaginaire, du culturel, de l'éthique et de l'axiologique dans une perspective esthétique.

4 Cette forme de recognition accompagne les actes ou les opérations de compréhension et d'explication au niveau de la réception. 
Riffaterre appelle « herméneutique ${ }^{2}$ » la lecture qui succède au premier contact avec le texte. En elle, le lecteur répare la mauvaise interprétation ou la première impression que le lecteur se fait lors de sa première réception du texte.

Il n'y a pas que le lecteur qui agisse ainsi, passant de l'interprétation à la mésinterprétation. Le scripteur procède en tissage et en mé-tissage si on peut nous passer l'expression, c'est-à-dire en raturant à merci les jeux d'hésitation, de perplexité, les représentations en silhouettes, les jugements hâtifs de la perception... Le terme de tissage n'est pas utilisé en tant que simple écho d'une généalogie suspendue ou dans le sens d'une métaphore délavée, mais c'est parce que le texte n'arrête pas d'user du champ lexical afférent à cette opération. Le narrateur rapporte que son maître Guillaume n'abandonnera pas cette affaire « quelle que fût la façon, il débrouillerait les fils de l'enquête $»^{3}$. Ce même narrateur rapporte le discours de son maître qui fait référence à ce dé-montage de l'enquête, en y associant le plaisir de la découverte qui consacre l'esprit :

«ce n'est pas de découvrir les coupables qui intéresse Bernard, mais de brûler les prévenus. Moi, par contre, je trouve mon plus grand plaisir, ma plus grande joie à démêler un bel écheveau bien enchevêtré. Et ce doit être encore parce que dans un moment où, comme philosophe, je doute que le monde ait un ordre, je trouve une consolation à découvrir, sinon un ordre, du moins une série de liens dans les menus lots des affaires du monde $»^{4}$

C'est dans cet esprit qu'Eco a conçu son premier roman en troquant sa serviette de sémioticien contre la plume du romancier. Habitué à traquer la signifiance et les processus sémiotiques du discours, l'auteur récidive, en empruntant le simulacre du détective percepteur philosophe. Si l'un cherche à éclaircir les mystères et les meurtres injustifiés et incompris qui choquent l'abbaye en passant de trace à trace ${ }^{5}$, c'est-à-dire de signe qui contient une référence au passé tout en prévoyant un futur (devenant indice), l'autre est en quête de rationalité, de cohérence et de logique ; le percepteur est entre les deux, car son discours, essentiellement didactique, semble allier les deux expériences dans les opérations de la compréhension et de l'explication. Ce syncrétisme actoriel justifie par ailleurs la démarche de la quête vers laquelle convergent les différentes attitudes susmentionnées en convoitant la véritéb. Or, qui dit vérité, dit sens caché. L'interaction entre la présence de quelque chose et son estompage dû à une force agissante est actualisée dans le roman à plus d'un endroit ; néanmoins, il y a un espace qui semble condenser à lui seul cette dimension : c'est celui de la bibliothèque. Cette dernière, mémoire de la chrétienté, est décrite en ces termes :

«Pour ces hommes voués à l'écriture, la bibliothèque était à la fois la Jérusalem céleste et un monde souterrain aux confins de la terre inconnue et des enfers. Ils étaient dominés par la bibliothèque, par ses promesses et par ses interdits. Ils vivaient avec elle, pour elle et peut-être contre elle, dans l'espoir coupable d'en violer un jour tous les secrets. Pourquoi n'auraient-ils pas dû risquer la mort pour satisfaire une curiosité de leur esprit, ou tuer pour empêcher que quelqu'un ne s'appropriât un de leurs secrets jalousement gardé ? " ${ }^{7}$.

Voulue pour garder les livres au lieu d'éclairer les esprits ${ }^{8}$, la bibliothèque, hermétique (dans tous les sens du terme, à savoir inviolable, secrète et obscure), double la rétention de l'information chez certains personnages du roman, mais aussi celui du vœu du silence des moines. Ce dernier, combiné à la dialectique de l'être et du non être du sens qui se dérobe et se trahit tout à la fois, justifie le titre du roman: Il nome della rosa, le Nom de la rose, interpellant implicitement la devise ecclésiastique sub rosa, c'est-à-dire littéralement sous la rose et par spécialisation sous le sceau du silence. 
9 La quête de la vérité qui se défile finit par ailleurs par orienter vers l'identité, dans le sens d'une conscience élevée de soi-même. C'est alors que l'enquête et l'aventure sémiotique deviennent une en-quête, une quête de l'être comme souci de vérité. En effet, décider de résoudre les mystères des meurtres en contournant les règles et aller à la recherche de preuves et d'indices entre dans la perspective d'une volonté de se prouver à soi-même, voire d'une sorte d'auto-gratification de l'homme excessivement fier de ses capacités intellectuelles, frisant peu ou prou le narcissisme. En outre, la décision de l'Abbé d'interdire toute recherche dans la bibliothèque a permis non seulement de révéler la ténacité de Guillaume, qui renforce sa volonté de poursuivre son enquête malgré tout, mais souligne aussi sa forte personnalité qui considère l'interdiction comme une atteinte à son intégrité scientifique :

«L'Abbé m'a signifié dès le début que la bibliothèque est intouchable [...] Et il ne veut pas qu'on découvre la vérité, ou du moins il ne veut pas que je la découvre moi... $»^{9}$.

10 Ce «moi » trahit l'idée que se fait le personnage de lui-même et transforme par conséquent l'enquête en une épreuve qui concourt aussi bien à résoudre le mystère des crimes commis dans ce «lieu abandonné de Dieu» qu'à glorifier l'ego savant et rationnel de Guillaume.

11 Ainsi, au fur et à mesure que le lecteur avance dans le texte, il assiste à sa gestation et à sa genèse. Le processus de transformation ne concerne pas seulement la transformation d'un état initial en situations intermédiaires pour retrouver un équilibre dans un état final, mais cela concerne aussi les incessants remaniements que se permet parfois le narrateur et d'autres fois Guillaume concernant les faits ou la réception que les personnages font des événements, dont ils sont contemporains et auxquels ils assistent. Ces changements d'optique présupposent le renvoi à une mémoire qui fonctionne comme thésaurus, une banque de données ou un inconscient, qui ne tarde pas à passer de l'estompement à l'activation de programmes périphériques. Le roman reproduit les structures mnésiques du fonctionnement cognitif humain, car le roman nous permet de comprendre après coup que la lecture, tout comme l'écriture d'une certaine manière, passe par la réactivation d'un certain nombre de représentations inscrites dans des conditions d'encodage, avec ce que cela présuppose de :

12 - modalités perceptives

- contraintes spatio-temporelles (par exemple enquêter la nuit dans un lieu où tout le monde se tait et où tout est interdit),

- l'état cognitif (enquêter dans un lieu hautement interdit, accompagné de beaucoup de prudence et de frayeur),

- motivations (la vérité/la justice, l'ego du savant et l'amour du savoir),

- ce qui est passé par une phase de rétention.

13 L'enquête policière ou le raisonnement logique de Guillaume mettront en œuvre l'opération de rétroaction par inférence, transfert, récupération des différents moyens d'accès à ce qui a été encodé, c'est-à-dire la mémoire du texte. Ainsi, quand Guillaume déchiffre le message de Venantius écrit en symboles zodiacaux, il ne parvient pas cependant à le comprendre; pour cela, le lecteur doit attendre la fin du roman pour voir le personnage réinterpréter «idolum» qui n'est pas l'idole, mais "l'image du miroir $»^{10}$, en se servant d'indices passés inaperçus, comme par exemple rappeler que l'auteur du message pensait en grec et non en latin. 

menant vers une orientation qui s'avère en fin de compte insignifiante, erronée ou inappropriée. C'est le procédé du divertissement dans le sens étymologique que prend ce terme, à savoir détourner du chemin et de la voie. Le lecteur consomme durant une grande partie de l'histoire l'interprétation du schéma des meurtres à partir de la surdétermination du livre de l'Apocalyps ${ }^{12}$. En fait, on apprend après que le meurtrier se sert de l'interprétation Apocalyptique comme camouflage qui éloigne du véritable meurtrier et du véritable lieu du crime. En tant que procédé d'égarement, le leurre justifie la réécriture et la re-figuration après l'opération de rature et de froissement que nécessite la destruction de la vision mésinterprétée. Advient ainsi la métamorphose du récit qui réorganise ses données en se servant de la mémoire qu'il autorise.

17 A ce moment-là interviennent la prolepse et le silence comme épisodes ou détails, sinon volontairement estompés, du moins passés inaperçus.

18 La prolepse peut se lire comme l'inscription d'un futur en promesse d'avènement, à advenir ; à l'image d'une bande annonce, sa fonction est d'ordre apéritif et surtout régulatrice, rappelant à l'ordre, avant l'heure, ce qui risque de déraper. Cette référentialité inscrit dans le roman la présence de ce qui n'a pas encore eu lieu; en d'autres termes, il est question d'une promesse de sens, de vérité et de lumière (selon qu'on se positionne dans l'attitude du détective, du philosophe ou du percepteur !) pour mieux voir dans ce lacis discursif.

19 Le silence quant à lui est polymorphe. Outre le silence du prêtre dont les lèvres sont scellées par le sacrement de la confession et le vœu du silence, il y a le silence de la peur qui, dans le récit, se traduit par le procédé rhétorique de la réticence; réticence à dire ce qui risque de nuire à soi et aux autres. Le silence dans ces acceptions s'ajoute bien évidemment à la thématique du secret et à l'hermétisme de la bibliothèque. Au niveau de la narration, cela crée des moments d'estompement, des blancs que l'enquêteur aura à combler dans son enquête par le repérage des faits ou le philosophe par son raisonnement. Il en est de même pour le silence en tant qu'incapacité à saisir le sens des signes interprétants qu'on rencontre et devant lesquels on passe sans leur prêter l'attention requise. C'est le cas où l'évidence devient énigme ou quand l'énigme offre sa résolution dans l'évidence.

20

Dans tous les cas, tout fonctionne dans ce roman selon le réseau d'associations schématiques $^{13}$, où le maître mot de l'affaire demeure la raison, la ratio, le raisonnement, la logique ${ }^{14}$ et l'herméneutique ${ }^{15}$. Dans cet esprit, entre tout le travail de l'écriture romanesque à mettre en scène les différentes variétés de l'argumentation ${ }^{16}$, parce que de ces types d'arguments dépend l'information, mais aussi la désinformation (au niveau de la signification), la formation et la déformation (au niveau de la structure), le plaisir et les rebondissements (au niveau de l'interprétation).

Cahiers de Narratologie, 33 | 2018 
21 Le grand Aristote, présent dans le roman par l'isotopie du rire, par l'esprit de la raison et par son livre, meurtrier hilare de cette histoire, a le mérite d'orienter la métamorphose du texte du côté de la métaphore. Plusieurs passages évoquent les possibles expansions imaginaires et sémantiques dont parle ce philosophe dont on cherche par tous les moyens à cacher son livre sur « le rire $»^{17}$. Le narrateur reproduit un passage du prétendu livre dont on peut citer un fragment: "dire les choses contraires à celles qu'on croyait, dire une chose et en entendre une autre ${ }^{18}$

Ici, il est question de la définition de la métaphore dans la mesure où les termes engagent une signification présente -absente par le biais d'une sorte d'agrammaticalité ou d'énoncé insensé, c'est-à-dire du côté de ce qu'on pourrait appeler l'autodestruction sémantique, dans la mesure où l'énoncé métaphorique est un énoncé impertinent qui ne signifie rien au niveau dénotatif et donc ne signifie qu'à condition d'y voir autre chose que ce qui est engagé par l'arrangement lexicosyntaxique. Ce sursis du sens de l'énoncé métaphorique a son importance, dans une situation où tout est affaire d'interprétation ${ }^{19}$ dans une situation où entrent en concurrence les « conflits d'interprétation ». Paul Ricoeur écrit dans cette optique :

«il est remarquable en effet que chez Aristote hermenêia ne se limite pas à l'allégorie, mais concerne tout discours signifiant; bien plus, c'est le discours signifiant qui est hermenêia, qui « interprète » la réalité, dans la mesure même où il dit « quelque chose de quelque chose »; il y a hermenêia, parce que l'énonciation est une saisie du réel par le moyen d'expressions signifiantes, et non un extrait de soidisant impressions venues des choses mêmes. $»^{20}$.

Ce qui nous intéresse ici en ce qui concerne la métaphore dans son rapport avec l'enquête, mais aussi la mise en valeur du raisonnement logique du philosophe, c'est le processus de décodage du sens. La métaphore selon Aristote revu par Ricoeur consiste dans le fait de représenter quelque chose selon le schéma suivant :

A est comme B sans être comme B

A est $B$ mais A n'est pas B

24 L'être passe par le truchement du non être, la vérité par celui de l'illusion, le sens passe par la fable. La question de l'interprétation est aussi une affaire d'argumentation comme le laissent entendre les procédés rhétoriques convoqués pour ce faire et le déploiement de tout un espace discursif qui favorise le débat sous ses formes: la dialectique et la controverse.

Dans un univers gouverné par le pouvoir ecclésiastique à tous les niveaux, culturel, politique et social, U. Eco dans Le Nom de la rose met en scène les pensées de l'époque qui coexistent dans et par le conflit: d'un côté un esprit religieux et de l'autre un esprit rationnel. La ratio est constamment confrontée aux différentes manifestations du règne théologique, manifestations en situation d'antagonisme virulent. De ce fait, la pensée logique se trouve impliquée dans cet univers dialogique et dialectique de la dispute, de la discussion et donc de l'argumentation avec les autres pensées, elles-mêmes fondées sur des arguments. Cette situation oblige l'esprit logique à se préciser de plus en plus comme discours de la démonstration visant l'universalité du raisonnement face à une argumentation qui ne se soucie que de persuader ou de convaincre selon les convictions du locuteur ou selon le vraisemblable.

Par ailleurs, si la pensée scolastique s'appuie sur l'enseignement des valeurs théologiques, la dimension didactique présente dans le roman n'est pas un élément du reflet historique, ou bien une surdétermination du genre oratoire en vogue à l'époque 
(où se déroulent les événements), mais plutôt une manière de justifier la variété des types d'argumentation par l'intermédiaire de Guillaume de Baskerville qui ne se lasse pas d'enseigner à son disciple, par des formes multiples, un certain savoir.

Guillaume apparaît dès l'ouverture du roman comme un personnage perspicace et sage. Il attire l'attention de son entourage par beaucoup de qualités comme le charme verbal et l'esprit sagace. Les événements vont prouver la description qu'en fait Adso, son disciple et narrateur du récit, dans la mesure où sa perspicacité ne l'absout pas d'émettre des réserves, soit par la perplexitée ${ }^{21}$, soit par la curiosité ${ }^{22}$ dont la probabilité logique autorise l'exercice ${ }^{23}$.

En effet, son entrée au monastère est triomphante, dans la mesure où il a réussi à orienter les chercheurs du cheval de l'Abbé, ayant remarqué sur son chemin les traces laissées par lui sur la neige. En répondant à l'accueil courtois du Cellérier :

«[... j'apprécie d'autant plus votre courtoisie que pour me saluer vous avez interrompu votre poursuite. Mais n'ayez crainte, le cheval est passé par ici et a pris le sentier de droite. Il ne pourra pas aller bien loin car, arrivé aux dépôts des litières, il devra s'arrêter. Il est trop intelligent pour se précipiter le long du terrain abrupt... $»^{24}$.

Guillaume a laissé croire au Cellérier que les voyageurs ont rencontré le cheval, mais la réplique négative du premier le surprend, d'autant plus que Guillaume renchérit en montrant connaître le nom du cheval :

" [...] il est évident que vous êtes en train de chercher Brunel, le cheval préféré de l'Abbé, le meilleur galopeur de votre écurie, avec sa robe noire, ses cinq pieds de haut, sa queue somptueuse, son sabot petit et rond mais au galop très régulier ; tête menue, oreilles étroites mais grands yeux. Il a pris à droite [...] $\aleph^{25}$

Il parvient en fait à en faire une description détaillée. Par ce discours, Guillaume cherche à «faire sensation » et à exporter vers le monastère, où sa renommée le précède, la réputation d'un homme au courant des secrets du type miraculeux; ce qui renforcera sa notoriété et son autorité morale, dans cet univers gouverné par le respect inconditionnel de cette forme de savoir.

31 En réalité, le personnage a procédé par abduction qui est, rappelons-le, un raisonnement à partir d'« inférences hypothétiques", c'est-à-dire une opération mentale "où le conséquent est supposé et suppose à son tour une Loi dont le conséquent serait alors le Cas, tout comme l'antécédent est le Résultat ${ }^{26}$. Le raisonnement de Guillaume en ce qui concerne l'affaire du cheval est de type abductif, mais la segmentation des traces, de leur implication et donc des étapes du raisonnement, démontrent qu'il y a également une opération de déduction. Il s'en explique à son disciple :

«Au croisement, sur la neige encore fraîche, se dessinaient avec grande clarté les empreintes des sabots d'un cheval, qui pointent vers le sentier à main gauche. $\mathrm{A}$ belle et égale distance l'un de l'autre, ces signes disaient que le sabot était petit et rond, et le galop d'une grande régularité - j'en déduisis ainsi la nature du cheval et le fait qu'il ne courait pas désordonnément comme fait un cheval emballé. Là où les pins formaient comme un appentis naturel, des branches avaient été fraîchement cassées juste à hauteur de cinq pieds. Un des buissons de mûres, là où l'animal doit avoir tourné pour enfiler le sentier à sa droite, alors qu'il secouait fièrement sa belle queue, retenait encore dans ses épines de longs crins de jais. [...] Si le cheval dont j'ai deviné le passage n'avait pas été vraiment le meilleur de l'écurie, on aurait peine à expliquer pourquoi ne le poursuivaient pas les seuls palefreniers, mais que se soit dérangé le Cellérier en personne ${ }^{27}$ 
On peut par ailleurs considérer la performance de Guillaume comme un fait de la dramatisation du récit, qui nous présente son héros par l'action. La compétence sousentendue sera prouvée à plusieurs reprises quand le personnage fait montre d'une aptitude à raisonner sans égale dans la mesure où elle est polymorphe.

En effet, Guillaume se sert par exemple de l'analogie pour provoquer Jorge :

«Mais alors je comprends maintenant pourquoi Venantius, au cours de cette conversation dont il me parlait hier, portait un tel intérêt aux problèmes de la comédie ; de fait, les fables de ce type-là aussi peuvent être assimilées aux comédies des antiques. L'une et l'autre ne racontent pas l'histoire d'hommes qui existèrent vraiment, comme les tragédies mais, dit Isidore, sont des fictions ${ }^{28}$.

4 Le recours à ce procédé est en fait un trait rhétorique courant au Moyen-âge et particulièrement la Scolastique où le logos se retrouve écarté au profit de l'ethos et du pathos ${ }^{29}$. C'est pour cette raison que, dans les écrits de l'époque, le mot "comme» revient souvent, suffisant à lui seul à inscrire dans l'énonciation une posture savante, utilisé par les hommes de religions comme par ceux de la science.

Dans un débat, on ne peut que recourir à l'analogie qui est, dans la majorité des cas, introduite par "comme $»^{30}$. Ce procédé se justifie du fait que, dans le cadre de sa conversation avec Jorge, Guillaume utilise les règles de la disputatio, à savoir l'échange de raisonnement contre raisonnement où souvent l'analogie pose une forme de réfutation quand on examine le discours des deux personnages. L'argumentation passe du raisonnement démonstratif à l'éloquence oratoire par la prolifération des figures de style / de rhétorique comme nous l'avons évoqué précédemment. Dans ce contexte aussi, la symétrie prend quelque fois l'aspect antithétique, notamment par l'utilisation du chiasme. Guillaume utilise également ce procédé pour détourner la conversation vers l'orientation que son interlocuteur cherche à esquiver et, de cette manière, le provoquer. Le chiasme se trouve ainsi associé, sinon à l'ironie, du moins à l'antiphrase. seulement l'irrite, mais surtout le désarme, parce que, ridiculisé, il perd le contrôle de sa pensée. L'argumentation ironique est donc une occasion qui permet au personnage de vider les propos de l'autre, de le désarmer, voire de le confondre au point de l'amener à sortir de sa réserve bien étudiée. Cela est manifeste aussi dans l'auto-ironie ou chleuasme. Ce procédé est une manière d'avoir le dessus sur un adversaire et de se faire connaître comme "héros» face au vaincu, se définissant désormais en antihéros, étant donné qu'il convoite le même objet de valeur, à savoir s'imposer à l'autre et à l'auditoire comme le plus convaincant et, par conséquent, le plus sage.

Par ailleurs, quand Guillaume prend la parole pour faire son discours devant les légats "d'une étrange conception $d u$ gouvernement temporel $»^{31}$, il développe un raisonnement logique certes! mais truffé d'arguments de notoriété, parce que l'auditoire est tellement convaincu par des a priori idéologiques qu'il est difficile, voire impossible, de persuader sans recourir à des auctoritates qui viennent, pour ainsi dire, au secours de la ratio et de Guillaume lui-même, c'est-à-dire lui évitent d'être taxé d'hérétique. Ainsi, il leur dit :

«Malheureux même, les infidèles [...] qui n'ont pas semblable autorité interprétant chacun pour soi la parole divine [...] Mais pouvons-nous dire pour autant, que les infidèles n'ont pas tendance à faire des lois et à administrer leurs affaires au moyen de gouvernements, rois, empereurs ou sultans et califes, comme on voudra? Et pourrait-on nier que de nombreux empereurs romains eussent exercé le pouvoir temporel avec sagesse, qu'on songeât à Trajan ? Et qui a donné, à des païens et à des 
infidèles, cette capacité naturelle à légiférer et de vivre en communautés politiques ? Leurs divinités mensongères peut-être, qui nécessairement n'existent pas (ou n'existent pas nécessairement, de quelque façon qu'on veuille entendre la négation de cette modalité) ? $»^{32}$. c'est-à-dire par le recours à un fait singulier, non général, qu'il soit réel ou imaginaire, mais surtout plausible. Dans les exemples de la citation susmentionnée, il est question de l'opinion de Guillaume en tant que savant reconnu par les légations ennemies, d'abord du fait même de la tâche dont il est investi par l'Empereur, à savoir réconcilier les deux délégations, ensuite par sa réputation d'homme sage et perspicace. Ainsi, les exemples associés à la démonstration garantissent le caractère convaincant de son discours. D'ailleurs, il n'a été réfuté par aucun des présents, en dépit de leur désaccord apparent. Ce qui prouve l'esprit astucieux du personnage.

En effet, Guillaume garde constamment son esprit en éveil ; et par intelligence et par respect de l'institution ecclésiastique, il fait passer son message " laïque » en s'appuyant sur des arguments religieux. Cependant, le vrai visage du personnage réside dans le cheminement de sa pensée rationnelle. Il apparaît dans les étapes de sa résolution du mystère des meurtres et de la bibliothèque ${ }^{33}$. Ce qui revient à dire que son portrait ne s'achève qu'à la fin du roman et, par conséquent, sa démonstration logique prend plus de six cents pages pour se dévoiler. Il définit lui-même sa démarche :

« résoudre un mystère n'est pas la même chose qu'une déduction à partir de principes premiers. Et ça n'équivaut pas non plus à recueillir une bonne quantité de données particulières pour en inférer ensuite une loi générale. Cela signifie plutôt se trouver en face d'une, ou deux, ou trois données particulières qui apparemment n'ont rien de commun, et chercher à imaginer si elles peuvent être autant de cas d'une loi générale que tu ne connais pas encore, et qui n'a peut-être jamais été énoncée ${ }^{34}$

En véritable philosophe du langage, Eco rappelle les deux démarches de la logique du raisonnement (d'abord la déduction, ensuite l'abduction), parce qu'il fait assumer à Guillaume dans le récit plusieurs rôles (émissaire de paix, percepteur, enquêteur, avocat) qui, à chaque situation diégétique, finissent par se rencontrer, dans la mesure où l'argumentation à travers le discours du personnage passe par le raisonnement : en essayant de trouver un consensus entre la délégation papale et celle des franciscains, en essayant de démêler les mystères des meurtres commis et celui de la bibliothèque, en défendant le Cellérier et son aide et en enseignant à son disciple. Cette variété de postures met en valeur l'évolution de la pensée, qui s'opère progressivement dans la période scolastique, mais aussi, l'ouverture d'esprit de Guillaume qui, en fonction des situations et des problèmes, passe de la diplomatie, à la philosophie, à la sémiotique et à la didactique. Loin d'être un jeu de rôles, c'est une filature du sens; or, il ne peut y avoir filature sans adaptation formelle au problème et à son contexte; c'est une adaptation méthodologique, voire paradigmatique dont les présupposés épistémologiques demeurent cependant les mêmes. C'est dans cet ordre que Guillaume, en bon didacticien, peut dire :

«la recherche des lois explicatives, dans les faits naturels, procède de façon tortueuse. Devant certains faits inexplicables, tu dois essayer d'imaginer un grand nombre de lois générales, dont tu ne perçois pas encore le rapport avec les faits qui te font problème : et tout à coup, dans le rapport soudain d'un résultat, un cas et une loi, se profile à tes yeux un raisonnement qui te semble plus convaincant que les autres. Tu essaies de l'appliquer à tous les cas semblables, à l'utiliser pour en tirer des prévisions, et tu découvres que tu avais deviné. Mais jusqu'à la fin, tu ne 
sauras jamais quels prédicats introduire dans ton raisonnement et lesquels laisser tomber. Et c'est ainsi que je procède maintenant. J'aligne quantité d'éléments décousus et je fabrique des hypothèses. Mais je dois en fabriquer beaucoup, et nombre de celles-ci sont si absurdes que j'aurais honte de te les dire. $»^{35}$.

41 La méthode présentée ici en termes abstraits va lui permettre de commenter l'épisode où il s'est fait connaître comme " héros ", c'est-à-dire de concrétiser pour son disciple sa théorie. Le lecteur comprend aussi que le personnage est en train de proposer une sorte de poétique de l'enquête qui justifiera la résolution du mystère, mais surtout des orientations de l'enquête.

Cette démarche par abduction nous oblige à distinguer dans le raisonnement de Guillaume deux types :

- le premier, concernant le déchiffrage du mystère, est une démonstration continue dont les faits sont incontestables. Elle se rapporte à l'enquête « policière » du héros.

- le deuxième est une argumentation, car elle s'appuie sur « des prémisses qui ne sont pas nécessairement prouvées, ou évidentes, mais simplement vraisemblables, c'est-à-dire admises par la plupart, ou par les gens compétents ${ }^{36}$. Ce type apparaît chez Guillaume ${ }^{37}$ quand il discute avec Jorge, avec l'Abbé et devant les légats.

44 Pour éviter la torture, cette manière de s'auto-accuser de la part des victimes est affichée par l'Inquisiteur comme un aveu de culpabilité. Ce qui crée au niveau du discours une sorte d'argumentation zéro. Cependant, Bernard Gui procède autrement quand il accuse le Cellérier au début de l'interrogatoire :

«Un serment! [...] voilà une autre preuve de ta malice! Tu veux jurer car tu sais que je sais que les hérétiques vaudois sont prêts à n'importe quelle ruse, et même la mort, plutôt que de jurer ! [...] tu cherches à me convaincre que tu n'es pas ce que tu es afin que je ne dise pas que tu es ce que tu es! Tu jures alors? Jure pour être absous [...] Et aussi tout serment sera une nouvelle preuve de ta culpabilité $! »^{38}$.

Ainsi, ce discours ne modifie guère le point de vue posé au départ par le locuteur, parce que sa conviction est imposée aux auditeurs, selon une démarche circulaire. Reprenant la rhétorique scolastique, le personnage parle tout seul comme pour se convaincre luimême, rappelant ainsi la sophistique que Platon a bien pris soin de récuser, parce que l'orateur sophiste parvient à « résoudre tous les problèmes " par le discours, défendant la chose et son contraire ${ }^{39}$, par référence au sophiste célèbre Hippias (mort vers 343), comme la figure de l'Inquisiteur dans le roman. indiscutables. En homme âgé, doté d'une mémoire fabuleuse, il est habitué à la questio. Cette dernière est une forme scolastique consistant dans :

«l'énoncé d'un problème [...] ; la production d'auctoritates qui inclinent à conclure en un sens, puis celles qui inclinent à conclure dans un autre; la solution du problème ; la réponse aux arguments qui allaient dans le sens qu'on a refusé - c'està-dire plus volontiers que la réfutation des «autorités", leur mise en place, moyennant les précisions et les distinctions convenables, dans le cadre de la solution fournie. $»^{40}$.

Dans l'avant dernier chapitre du septième jour, Jorge se lance dans un débat à propos des ravages que cause la philosophie d'Aristote pour justifier ainsi ses actes. Le personnage use dans cette situation de paralogisme dans toute sa diversité d'enthymème apparent ou tronqué (c'est-à-dire où toutes les étapes du raisonnement ne sont pas démontrées, voire posées) ou bien en « réunissant ce qui est séparé ou en séparant ce qui est 
uni [...] Dans tous les cas, le paralogisme consiste à faire comme s'il y avait homologie ontologique ou existentielle entre l'ensemble et les éléments » ${ }^{41}$.

Chez Jorge, le paralogisme consiste également à passer à l'exagération de l'horreur du fait contesté, par le procédé de l'amplification systématique, cherchant à ébranler la conviction de Guillaume son interlocuteur, alors qu'il n'a pas donné les preuves de ce qu'il avance. Une analyse détaillée de ce discours révélera d'autres aspects du paralogisme, mais ce qui importe c'est que Jorge accompagne son argumentation de "sophismes ", c'est-à-dire de paroles sans argument ou évidence, ce qui la détermine comme perverse. Umberto Eco met en discours ce système d'argumentation scolastique pour démontrer comment la signification d'une proposition implique à la fois la rationalité logique, parce que cette dernière s'intéresse à ce qui est réel ou fictif, et à la rationalité ontologique dans la mesure où il pose la question de l'existence. C'est ce que la scolastique appelle la suppositio.

La lecture que nous faisons de ces quelques aspects de l'argumentation dans le roman Le Nom de la rose nous oblige à tirer des conclusions sur la finalité de cette dimension discursive, et à nous interroger encore sur les frontières entre ce qui, dans un texte, est argumentatif et ce qui ne l'est pas.

Quand l'Abbé mandate Guillaume pour enquêter sur "les événements terribles » du monastère, il attend de lui qu'il démêle les fils de cette affaire compliquée, mais aussi qu'il fasse preuve de sagesse en ne divulguant pas ses résultats. On remarque ici, quoique timidement, une distinction entre la nécessité de comprendre et l'évacuation de l'urgence d'expliquer, alors que la pensée médiévale est au croisement de ces deux notions appliquées bien entendu au texte sacré.

Dans son enquête "policière ", où la logique détermine le raisonnement, la trace (en tant que signe, empreinte ou marque conduisant à d'autres), laissée par la victime ou le meurtrier, devient l'argument de la démonstration qui écarte, plus ou moins, l'interprétation de l'intervention diabolique et maléfique. La trace invite à remonter le processus des faits, Eco dirait des théories, et à rechercher le sens tout comme le criminel. C'est donc une construction de données éparses ${ }^{42}$ par l'accumulation de preuves et de leur concordance ${ }^{43}$, y compris par l'absence de trace qui se signale comme pertinente par son absence même. C'est alors une invitation à raisonner. Or, il convient d'interroger cette pluralité argumentative à la lumière des modes de vision, parce que le roman Le Nom de la rose est également une affaire d'interprétation par son contexte médiéval et scolastique, mais surtout par son déploiement discursif entièrement construit sur des raisonnements antagonistes, dialectiques ou alternatifs. En outre, l'interprétation est le propre de la théologie, qui distingue dans le texte le niveau patent accessible aux profanes et le niveau latent réservé aux seuls initiés, conservateurs et promoteurs de la vérité et de la symbolique chrétienne.

Enfin, ce roman met en évidence la question de l'esthétique moderne, à savoir la dissimulation, voire la rature des zones spécifiques quant aux catégories génériques de l'écriture, en instaurant à la place un espace où règne une pluralité des voix qui le définit par la même occasion. Et nous pouvons nous interroger alors sur comment la narrativité s'institue-t-elle en tant que discours, en précisant néanmoins que, dans ce dernier, domine en priorité l'argumentation, au moment où le champ narratif c'est plutôt comme processus événementiel et action de raconter qu'il s'impose? 


\section{NOTES}

2. «Au fur et à mesure de son avancée au fil du texte, le lecteur se souvient de ce qu'il vient de lire et modifie la compréhension qu'il en a eue en fonction de ce qu'il est en train de décoder. Tout au long de sa lecture, il réexamine et révise, par comparaison avec ce qui précède. En fait, il pratique un décodage structural : sa lecture du texte l'amène à reconnaître, à force de comparer, ou simplement parce qu'il a maintenant les moyens de les assembler, que des éléments du discours successifs et distincts, d'abord notés comme de simples agrammaticalités, sont en fait équivalents puisqu'ils apparaissent comme les variants de la même matrice structurale. Le texte est donc une variation ou une modulation d'une seule structure - thématique, symbolique, qu'importe - et cette relation continue à une seule structure constitue la signifiance. ", Sémiotique de la poésie, Paris, Ed. Seuil, 1983, p. 17.

3. Umberto Eco, Le Nom de la rose, Paris, Ed. Grasset \& Fasquelle, coll. « Livre de poche », 1982, p. 174.

4. Ibid. p. 496.

5. Sur l'interprétation de la trace, on peut lire le passage du roman où le personnage de Guillaume tout en expliquant à son disciple comment il a réussi à connaître un cheval donné sans le voir, développe une véritable théorie du signe : " quand vous avez lu les traces sur la neige et sur les branches, vous ne connaissiez pas encore Brunel. D'une certaine manière, ces traces nous parlaient de tous les chevaux de cette espèce.ne faut-il donc point dire que le livre de la nature nous parle seulement par essences, comme enseignent moult éminents théologiens? [...] l'empreinte en ce lieu précis et à cette heure du jour me disait qu'au moins un cheval, parmi tous les chevaux possibles, était passé par là. Si bien que je me trouvais à mi-chemin entre l'acquisition du concept de cheval et la connaissance d'un cheval individuel. Et en tout cas ce que je savais du cheval universel m'était donné par la trace, qui était singulière. Je pourrais dire qu'à ce moment-là j'étais prisonnier entre la singularité de la trace et mon ignorance, qui prenait la forme extrêmement diaphane d'une idée universelle.", Ibid. pp. 41-42. Lire également la page 138 où il est question du signe qui conduit vers un autre signe.

6. Le roman développe toute une réflexion sur «la vérité », parce qu'elle concerne en premier lieu la rencontre organisée à l'abbaye entre l'ordre des franciscains et la délégation papale à propos de la pauvreté du Christ, rencontre dans laquelle Guillaume de Baskerville est mandaté pour tenter de réconcilier les deux délégations; en second lieu elle intervient dans le débat entre Guillaume et son disciple Adso à propos de la jeune fille accusé injustement par Bernard Gui l'Inquisiteur.

7. Ibid. p.232.

8. Cette attitude est incarnée par Jorge, esprit de l'abbaye et de la bibliothèque qui refuse à l'homme le droit à la pensée, car selon lui elle le détourne de la prière et de Dieu, contrairement de Guillaume.

9. Ibid. p.197.

10. Respectivement p. 263 et p.402.

11. Cf. S/Z, Paris, Editions du Seuil, « Points », 19

12. cf. p. 201 et p. 460.

13. Il est question ici de toute forme de schématisation qui entre dans la mise en place d'une sorte de structure de connaissance par prototype. Cf. l'ouvrage collectif, Traité de psychologie cognitive, «Traitement de l'information symbolique », Paris, Dunod, 1990.

14. Cf. p. 330 où Guillaume enseigne à son disciple les types de raisonnement et le prévient que ces procédés ne sont pas infaillibles. 
15. Beaucoup de passages traitent de la compréhension et de son corollaire l'interprétation. On peut se référer par exemple à l'interprétation du message de Venantius où il est question de symboles. Paul Ricœur dans sa perspicacité habituelle note que l'interprétation renouvelle les significations bien installées dans une tradition en faisant appel «à sa réserve de sens ». Il ajoute : «L'interprétation de la symbolique ne mérite d'être appelée herméneutique que dans la mesure où elle est un segment de la compréhension de soi-même et de la compréhension de l'être ", Les conflits d'interprétation, Paris, Ed. Seuil, 1969, p.33.

16. Le roman d'Eco met en discours une variété de modes d'argumentation: induction, abduction, déduction, paralogisme, enthymème, argument de notoriété, l'exemple... Pour plus de précision, cf. notre article "Le Nom de la rose ou l'argumentation polymorphe ", actes du colloque international qui s'est déroulé à Fès les 7 et 8 mars 2002, Discurso y argumentacion, Fès, Publications de la Faculté des Lettres et des sciences humaines Dhar El Mahraz Fès, 2005, pp. 199-212.

17. Cf. p. 110, p. 166.

18. P. 358.

19. A la page $211 \mathrm{du}$ roman, on peut lire par exemple: «On peut cependant formuler des hypothèses sur les mots qui pourraient être les premiers du message, et ensuite voir si la règle qu'on en infère vaut pour tout le reste de l'écrit. » La suite du dialogue montre les étapes de l'interprétation.

20. Les conflits d'interprétation, op. cit. p. 8.

21. Cette perplexité va permettre au personnage de la développer sous forme théorique dans le concept de la mésinterprétation. En réalité, cette mésinterprétation va être mise en pratique parce que Guillaume vérifie toujours ses hypothèses dans son enquête, occasion pour enseigner à son disciple que l'argumentation ne conduit pas toujours à la vérité, et surtout pour relancer l'histoire. Par ailleurs, la perplexité est une forme d'argumentation du type cognitif.

22. La curiosité nous rappelle, en plus de ce qu'elle évoque de la technique des détectives, à savoir le flair, ce qu'Eco nommera dans les Limites de l'interprétation « le syndrome du doute ».

23. Cf. Le Nom de la rose, Paris, Editions Grasset \& Fasquelle, «Livre de poche », 1982, pp. 186, 178, 210. Toutes les citations du roman renvoient à cette édition.

24. Ibid. p. 35.

25. Ibid.

26. Umberto Eco, Sémiotique et philosophie du langage, Paris, PUF, « Formes sémiotiques », 1988, p. 47.

27. Pp. 36-37.

28. P. 167.

29. Le rejet du logos n'est en fait qu'une marginalisation par la domination des deux catégories (ethos et pathos) du fait de la nature du discours de l'époque, entièrement déterminé par l'élite politique au pouvoir et surtout par les théologiens qui prisaient les arguments d'autorité. Ainsi la dialectique va finir par devenir une controverse, c'est-à-dire une disputatio, une discussion de "sourds", donc une dispute du moment qu'on n'entend que ses propres arguments, rejetant ceux des autres. C'est ce type de débat qui va affaiblir l'argumentation et écarter le logos. Néanmoins, l'ethos va prendre le devant de la scène rhétorique parce qu'il est centré sur l'orateur qui est présupposé incarner un schème bien défini socialement au niveau des valeurs et de la vision du monde de la communauté à laquelle on appartient; bref, ce à quoi une époque s'identifie. A côté de ce genre, le pathos en tant que discours prend en charge les passions de l'auditoire (le récepteur) en transformant à la fois la question de la situation en cours (problème qu'on est en train de débattre ou, en termes plus contemporains, la problématique posée) et les attentes de cet auditoire en une sorte de réponse, ou plutôt en une réflexion-réponse, un discours qui présente le problème en termes de solution. D'où le recours aux figures d'ornement en vigueur chez l'élite religieuse (le Vatican) et politique (les cours des monarques). 
30. " Je crois que le rire est une bonne médecine, comme les bains, pour soigner les humeurs et les autres affections du corps, en particulier la mélancolie », p. 50.

31. A titre d'exemple : « on ne tue pas sans raison, pour perverse qu'elle soit. Et je tremble de la pensée de la perversité des raisons qui peuvent avoir poussé un moine à tuer un confrère », p. 440.

32. P. 446.

33. Le roman tourne autour de la Bibliothèque qui assure plusieurs fonctions dans le récit: elle est à la fois un espace, une allégorie du savoir et du labyrinthe.

34. P. 383.

35. P. 384 .

36. Olivier Reboul, La Rhétorique, Paris, PUF, « Que sais-je ?», 1993, p. 66.

37. Néanmoins, l'on peut dire que c'est le premier type qui domine chez le personnage, parce qu'il correspond à la nature du héros, soit en enseignant à Adso les choses du monde et de la religion, soit dans son approche des événements qui ont secoué le monastère. Parallèlement à la manière de raisonner de Guillaume, le roman met en relief d'autres modes, notamment celui de l'Inquisition dans le personnage de Bernard Gui et celui de l'extrémisme religieux de Jorge. En ce qui concerne Bernard Gui, c'est un personnage qui joue plus sur la terreur, qu'inspire sa fonction sur ses victimes et sur les moines, que sur une vraie argumentation. Souvent son discours ne développe aucun argument, se limitant à accuser violemment sa victime et l'obligeant à avouer des crimes qu'elle n'a pas commis, en répétant tout simplement ce qu'il lui dit. Ainsi, quand le Cellérier accusé dit : « Mais que dois-je avouer?». Bernard Gui répond : « Deux ordres de péchés. Que tu as été de la secte de Dolcino, que tu en as partagé les propositions hérétiques [...] » p. 481.

38. P. 480.

39. Lucien Jerphagnon se penche en détail sur la question dans son livre Histoire de la pensée, « D’Homère à Jeanne d'Arc », Editions Thaillandier, « Pluriel », 2009, PP. 80-91.

40. Article «Scolastique », Encyclopedia Universalis, vol. 14, p. 774.

41. Georges Molinié, Dictionnaire de rhétorique, Paris, Livre de Poche, 1992, p. 243. C'est ainsi que le personnage de Jorge peut dire : « Au moment où il rit, peu importe au vilain de mourir [...] Et de ce livre pourrait naître la nouvelle et destructrice aspiration à détruire la mort à travers l'affranchissement de la peur. », 593.

42. Cf. p. 177.

43. Cf. p. 263.

\section{ABSTRACTS}

This article tries to study the mobilization of two registers of writing in the Name of the rose: the didactic register and the police register. The first being characterized by linearity, truth and the second by the erasure, hesitation and trampling. These two seemingly opposite registers, complement each other in a masterful way in the novel and constitute the spring that makes the characters, the plot and the commentaries of the narrator work. The article is not content to describe how this double register makes the novel turn, but is inspired by it to deliver impressions on the two modes of writing.

Cet article essaie d'étudier la mobilisation de deux registres d'écriture dans le Nom de la rose : le registre didactique et le registre policier. Le premier étant caractérisé par la linéarité, la vérité et 
le second par la rature, l'hésitation et le piétinement. Ces deux registres en apparence opposés, se complètent de façon magistrale dans le roman et constitue le ressort qui fait fonctionner les personnages, l'intrigue et les commentaires du narrateur. L'article ne se contente pas de décrire comment ce double registre fait tourner le roman, mais s'en inspire pour livrer des impressions sur les deux modes d'écriture.

\section{INDEX}

Keywords: Eco, Name of the rose, didactic register, investigation

Mots-clés: Eco, Nom de la rose, registre didactique, enquête

\section{AUTHOR}

KHALID HADJI

Laboratoire LaRELALaboratoire de Recherche sur l'Expression Littéraire et Artistique, Faculté des Lettres et des Sciences Humaines Dhar El Mahraz, Université Sidi Mohamed Ben Abdellah, Fès, Maroc, Université de Fès 